University of Nebraska - Lincoln

DigitalCommons@University of Nebraska - Lincoln

Shireen Adenwalla Papers

Research Papers in Physics and Astronomy

2018

SAW assisted domain wall motion in Co/Pt multilayers

Westin Edrington

Uday Singh

Maya Abo Dominguez

James Rehwaldt Alexander

Rabindra Nepal

See next page for additional authors

Follow this and additional works at: https://digitalcommons.unl.edu/physicsadenwalla

Part of the Condensed Matter Physics Commons, Engineering Physics Commons, Materials Science and Engineering Commons, and the Nanoscience and Nanotechnology Commons

This Article is brought to you for free and open access by the Research Papers in Physics and Astronomy at DigitalCommons@University of Nebraska - Lincoln. It has been accepted for inclusion in Shireen Adenwalla Papers by an authorized administrator of DigitalCommons@University of Nebraska - Lincoln. 


\section{Authors}

Westin Edrington, Uday Singh, Maya Abo Dominguez, James Rehwaldt Alexander, Rabindra Nepal, and Shireen Adenwalla 


\title{
SAW assisted domain wall motion in Co/Pt multilayers
}

\author{
Westin Edrington, ${ }^{1, a)}$ Uday Singh, $\left.{ }^{1, b}\right)$ Maya Abo Dominguez, ${ }^{1,2, c)}$ \\ James Rehwaldt Alexander, ${ }^{1,2, d)}$ Rabindra Nepal, ${ }^{1}$ and S. Adenwalla ${ }^{1}$ \\ ${ }^{1}$ Department of Physics and Astronomy and Nebraska Center for Materials and Nanoscience, \\ University of Nebraska-Lincoln, Lincoln, Nebraska 68588, USA \\ ${ }^{2}$ Lincoln High School, $2229 \mathrm{~J} \mathrm{St}$, Lincoln, Nebraska 68510, USA
}

(Received 13 August 2017; accepted 14 January 2018; published online 29 January 2018)

\begin{abstract}
The motion of domain walls in thin ferromagnetic films is of both fundamental and technological interest. In particular, the ability to use drivers other than magnetic fields to control the positions of domain walls could be exciting for memory applications. Here, we show that high frequency dynamic strain produced by surface acoustic waves is an efficient driver of magnetic domain walls in ferromagnetic films with perpendicular anisotropy. A standing surface acoustic wave of resonant frequency $96.6 \mathrm{MHz}$ increases the domain wall velocities in thin films of $[\mathrm{Co} / \mathrm{Pt}]_{\mathrm{n}}$ by an order of magnitude compared to magnetic fields alone. This effect is highly resonant, effectively ruling out thermal effects, and the velocity shows distinct variations in the domain wall velocity at the nodes and antinodes of the standing wave. The data indicate that standing strain waves can drive the domain wall motion from the creep to the flow regime as the amplitude increases. Hence, strain waves could provide an alternative route to rapid domain wall motion. Published by AIP Publishing. https://doi.org/10.1063/1.5000080
\end{abstract}

The motion of domain walls in thin film ferromagnets is an intriguing and complex puzzle ${ }^{1,2}$ with direct applications to magnetic memory technology. Racetrack memories ${ }^{3}$ are the blue ribbon winners when it comes to domain wall velocities, with speeds approaching $750 \mathrm{~m} / \mathrm{s}$ albeit at extremely high current densities of $10^{12} \mathrm{~A} / \mathrm{m}^{2}$. Domain walls driven by magnetic fields, a more conventional approach, exhibit slow domain wall velocities in the low field creep regime and fast motion at higher fields, the flow regime, with the crossover between the "high" and "low" field regimes dependent on extrinsic effects, such as the density and strength of pinning/ depinning sites. The complexity of domain wall motion has to do with the complex pinning/depinning energy landscape they traverse, and several publications have explored these models in some detail.

Here, we investigate domain nucleation and propagation in a $\mathrm{Co} / \mathrm{Pt}$ multilayer with perpendicular anisotropy in the presence of a surface acoustic wave (SAW). A recent paper that explored the response of domain walls to standing strain waves, using micromagnetic simulations, showed periodic domain wall velocities, corresponding to rapid motion and pinning at the nodes and antinodes, respectively. ${ }^{4}$ Our measurements indicate that the fast dynamic strain generated by SAW leads to a large increase in domain wall velocity. In addition, the increase in velocity is spatially periodic, with a pattern that is consistent with the standing wave generated by the SAW.

Two sets of interdigitated transducer (IDT) electrodes, each consisting of 27 finger pairs, were patterned on either

\footnotetext{
a) Present address: MOBI, Indianapolis, IN 46278, USA.

b) Present address: X-Wave Innovations, Inc., Gaithersburg, MD 20878 , USA.

${ }^{c}$ Present address: Bethesda-Chevy Chase High School, Bethesda, MD 20814, USA.

${ }^{d)}$ Present address: Brown University, Providence, RI 02912, USA.
}

side of a perpendicular anisotropy $\operatorname{Pt}(20 \AA) /[\operatorname{Co}(3 \AA) / \operatorname{Pt}(6 \AA)]_{5}$ multilayered thin film of dimensions $290 \mu \mathrm{m} \times 200 \mu \mathrm{m}$, all grown on $128^{\circ} \mathrm{Y}$-cut $\mathrm{LiNbO}_{3}$. (See Fig. 1). Both the IDTs and the $\mathrm{Co} / \mathrm{Pt}$ pad were patterned using photolithography followed by metallization with $\operatorname{Pt}(20 \AA) /[\operatorname{Co}(3 \AA) / \operatorname{Pt}(6 \AA)]_{5}$ and subsequent lift-off. The growth rate, power, and other sputtering parameters for $\mathrm{Co}$ and $\mathrm{Pt}$ are similar to those reported in Ref. 5.

The IDT finger widths and spacing resulted in a SAW wavelength $(\lambda)$ of $40 \mu \mathrm{m}$ and a resonance frequency of $96.6 \mathrm{MHz}$, with SAW propagation along the crystallographic $\mathrm{x}$-direction of the substrate, ${ }^{1}$ denoted by the $\mathrm{x}$-axis in Fig. 1 . The separation between the innermost fingers of the two sets of IDTs was chosen to be an integer number of wavelengths, $400 \mu \mathrm{m}$, resulting in the formation of a standing strain wave when both transducers are excited in phase.

The application of voltages to the IDTs generates both longitudinal $\varepsilon_{\mathrm{xx}}$ and shear strains $\varepsilon_{\mathrm{xy}}$ and $\varepsilon_{\mathrm{xz}}$, where the subscripts refer to the coordinate system of the sample as depicted in Fig. 1, not the crystallographic axes, with the shear $\varepsilon_{\mathrm{xy}}$ and longitudinal strain $\varepsilon_{\mathrm{xx}}$ being out of phase by $\pi / 2$. The effective magneto-elastic free energy is given by

$$
\mathrm{U}_{\mathrm{ME}}=\mathrm{B}_{1} \varepsilon_{\mathrm{xx}} \alpha_{\mathrm{x}}^{2}+\mathrm{B}_{2}\left(\varepsilon_{\mathrm{xy}} \alpha_{\mathrm{x}} \alpha_{\mathrm{y}}+\varepsilon_{\mathrm{xz}} \alpha_{\mathrm{x}} \alpha_{\mathrm{z}}\right),
$$

where the relevant magneto-strictive constants for fcc Co are $\mathrm{B}_{1}=-16 \times 10^{6} \mathrm{~N} / \mathrm{m}^{2}$ and $\mathrm{B}_{2}=26 \times 10^{6} \mathrm{~N} / \mathrm{m}^{2}$ and $\alpha_{\mathrm{i}}$ are the direction cosines of the magnetization. ${ }^{6}$ The out-of-plane magnetization results in $\alpha_{x}=0$ everywhere except at the domain walls between up and down magnetization. Previous experiments and micromagnetic simulations of domain walls in $\mathrm{Co} / \mathrm{Pt}$ multilayers and other thin films with perpendicular anisotropy indicate $e^{7,8}$ the existence of Bloch type domain walls at the film center (in which $\alpha_{\mathrm{x}}=0$ through the entire domain wall) transitioning to Neel type domain wall caps at the surfaces, in which $\alpha_{\mathrm{x}}=1$ at the center of the domain 

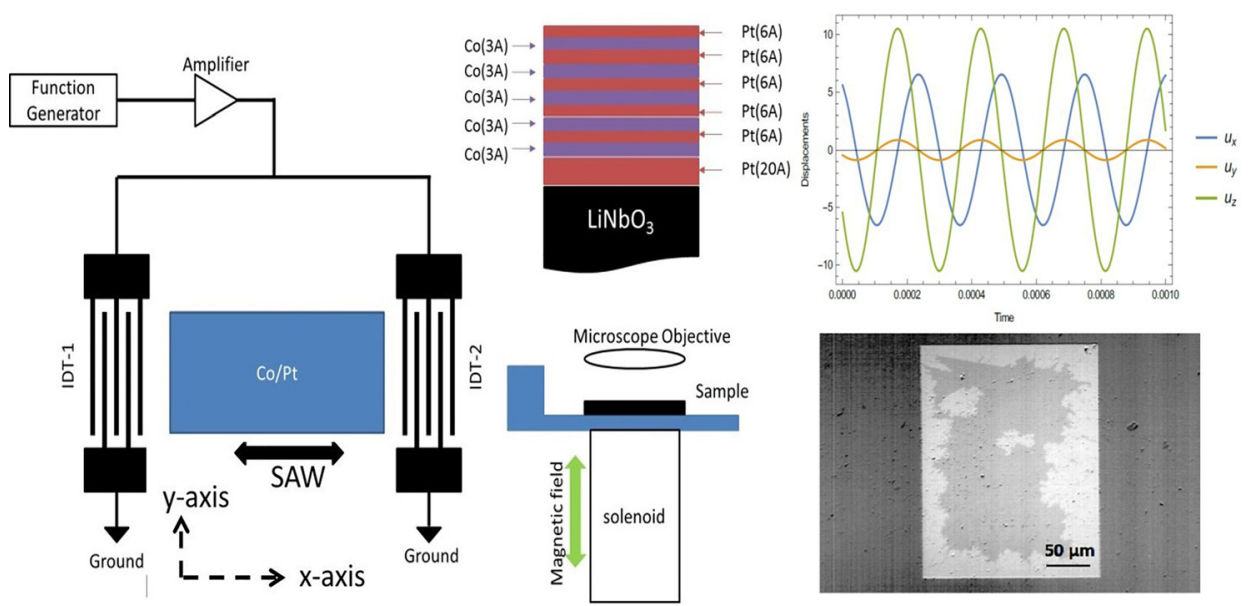

FIG. 1. A schematic of the sample with interdigital (IDT) electrodes and Co/Pt multilayers patterned on $128^{\circ}$ Y-cut $\mathrm{LiNbO}_{3}$. Each set of IDTs comprised 27 finger pairs, with a $10 \mu \mathrm{m}$ width and spacing, and SAW propagation along the x-axis. The two sets were separated by $10 \mathrm{SAW}$ wavelengths (400 $\mu \mathrm{m})$. A $290 \mu \mathrm{m}$ by $200 \mu \mathrm{m}$ rectangular pad of $\mathrm{Pt}(20 \mathrm{~A}) /[\mathrm{Co}(0.3 \mathrm{~nm}) / \mathrm{Pt}(0.6 \mathrm{~nm})]_{\mathrm{x} 5}$ was deposited between the two IDTs. The sample was mounted on an Al base with a solenoid positioned directly beneath the Co/Pt multilayer. Inset: (top left) The layer structure of $\mathrm{Co} / \mathrm{Pt}$ multilayers and (bottom left) the mounting scheme of the sample. (top right) The displacements, $\mathrm{u}_{\mathrm{x}}$ (blue), $\mathrm{u}_{\mathrm{y}}$ (red), and $\mathrm{u}_{\mathrm{z}}$ (green), as functions of time, showing the $\lambda / 4$ phase difference between the displacements $\mathrm{u}_{\mathrm{x}}$ and $\mathrm{u}_{\mathrm{y}}$. (bottom right) The MOKE image of the magnetic domains in the $\mathrm{Co} / \mathrm{Pt}$ multilayers.

wall. Hence, the effects of longitudinal strain, dependent only on $\alpha_{\mathrm{x}}$, will be highest at the Neel type end caps. In contrast, $\alpha_{\mathrm{y}}$ will only be non-zero in Bloch walls so that the effects of transverse strain, $\varepsilon_{\mathrm{xy}}$, will be non-zero only over the regions where the Neel wall transitions to a Bloch wall.

The relative values of the strain, $\varepsilon_{x x} / \varepsilon_{x y} \sim 20$ [inset of Fig. 1 (top right)], would indicate that the dominant strain term is $\mathrm{B}_{1} \varepsilon_{\mathrm{xx}} \alpha_{\mathrm{x}}^{2}$, and hence, the effective instantaneous pressure on a domain wall is $B_{1} \frac{d \varepsilon_{x x}}{d x} \Delta$, where $\Delta$ is the domain wall width. However, the larger value of $\mathrm{B}_{2}$ (compared to $\mathrm{B}_{1}$ ) and the extent of the transition region may increase the significance of the transverse strain in regions where the longitudinal strain gradient is small (the antinodes) because the $\pi / 2$ phase difference implies that $\varepsilon_{\mathrm{xx}}$ and $\varepsilon_{\mathrm{xy}}$ standing waves are shifted with respect to each other, viz. $2 \varepsilon_{\mathrm{xx}} \operatorname{Sin}(\mathrm{kx}) \operatorname{Cos}(\omega \mathrm{t})$ for the longitudinal standing wave vs. $2 \varepsilon_{\mathrm{xy}} \operatorname{Cos}(\mathrm{kx}) \operatorname{Cos}(\omega \mathrm{t})$ for the transverse wave. Hence, the pattern of nodes and antinodes for the longitudinal and transverse standing waves is shifted by $\lambda / 4$, corresponding to a spacing of $10 \mu \mathrm{m}$.

A white light magneto-optical Kerr effect (MOKE) microscope was used to image magnetic domains in the $\mathrm{Co} / \mathrm{Pt}$ rectangle and record their response to both the magnetic field and the SAW excitation. A $480 \times 640$ pixel COMS camera (Celestron) with a $10 \times$ objective lens resulted in a spatial resolution per pixel of $\sim 1.5 \times 1.5 \mu \mathrm{m}^{2}$. The sample was mounted on a homemade sample stage with the necessary rf connections and an iron core electromagnet positioned directly under the sample supplying an out-of-plane magnetic field. The two IDTs were connected in parallel to an rf signal generator (SRS SG380) and a power amplifier (Pasternak PE 15A4017) and were excited with $\mathrm{rf}$ input powers ranging from $-85 \mathrm{dBm}$ to $30 \mathrm{dBm}$. Both the magnetic field and the SAW were used to drive domain walls, which move in both the $\mathrm{x}$ and $\mathrm{y}$ directions (see S-2, supplementary material). However, because we are most interested in the effects of the SAW, we focus on $\mathrm{x}$-axis motion along the direction of SAW propagation.

The frequency response of the two IDTs was measured using a spectrum analyzer (Agilent E4407B), an input power of $-10 \mathrm{dBm}$, and a bidirectional coupler (Minicircuits ZFBDC20-62HP+). As seen in Fig. 2(a), the reflected power dips sharply at the resonance, where conversion of electrical to mechanical power is most efficient.

Our measurements include both spatially resolved pixelby-pixel measurements and spatially averaged measurements. The pixel-by-pixel MOKE domain images captured by the camera were acquired first at magnetic saturation, and these images were subtracted from subsequent images that were acquired with a series of one second long pulses of a reversed field. The domains were nucleated using a reverse field pulse of $134 \mathrm{G}$ (just above the coercive field) and subsequently moved using $1 \mathrm{~s}$ pulses close to, but just below, the coercive field. 200 images were averaged after each field pulse. The magnetic domains only move during the duration of the pulse, remaining steady in the interim. Image processing, including contrast enhancement and edge smoothing,

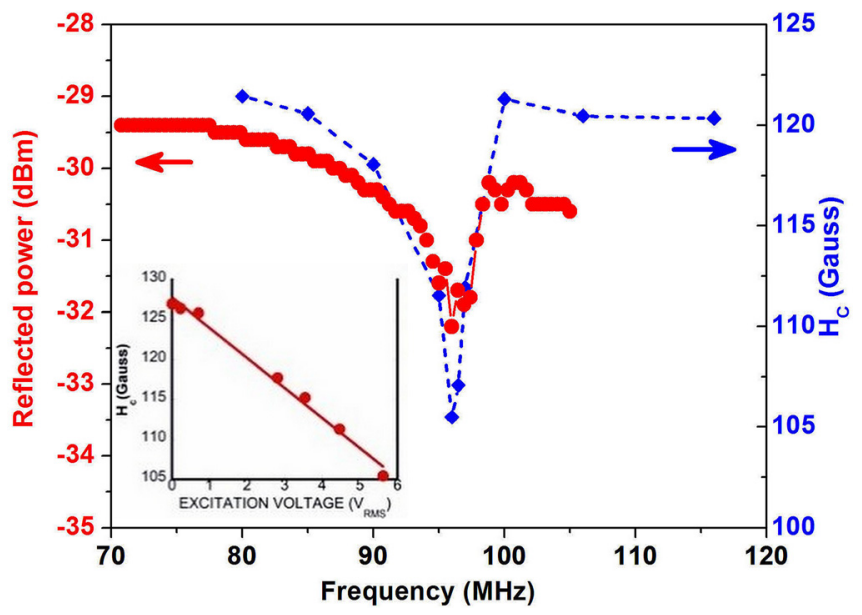

FIG. 2. The electrical response of the two IDTs (red), and the coercivity (blue) as a function of IDT excitation frequency. There is a sharp reduction in reflected power at the resonance frequency of $96.6 \mathrm{MHz}$, and the coercivity decreases sharply at the resonance frequency. Inset: Coercive field as a function of excitation voltage at the resonance frequency together with a linear fit. 
was carried out using Image J. ${ }^{9}$ The average velocity was obtained by averaging over the y-direction to obtain an average $\mathrm{x}$-position of the domain wall after every pulse. The slope of this average $\mathrm{x}$-position vs. pulse gives us the average domain velocity in the $\mathrm{x}$-direction. The spatial resolution and the constraints on the duration of the field pulse result in a lower limit on a domain wall velocity of $1.5 \mu \mathrm{m} / \mathrm{s}$, with the upper limit proscribed by the dimensions of the sample, $290 \mu \mathrm{m} / \mathrm{s}$. We note that we have achieved velocities at the upper limit, albeit with fairly large error bars, since we cannot average the velocity over subsequent field pulses. We have also performed continuous measurements (which preclude the image averaging procedure described above), in which the domain images are monitored as a function of time under the influence of a constant magnetic field, with and without the SAW.

Measurements of the domain velocity as a function of magnetic field pulse height (in the absence of SAW) are shown in the supplementary material, Fig. S-1. The error bars indicate the distribution of velocities, which occur due to pinning centers that are randomly scattered within the thin film. Over this limited range of fields, the average domain velocity shows the expected low field behavior, in the creep, rather than the flow, regime. ${ }^{10}$ Because our data were taken at fields very close to $\mathrm{H}_{\mathrm{c}}$, the domain velocities we report are much lower than those previously reported in similar $\mathrm{Co} / \mathrm{Pt}$ films. ${ }^{10,11}$ A fit to the creep equation $v=v_{0} \exp \left[\frac{-U_{c}}{k_{B T}}\left(\frac{H_{d e p}}{H}\right)^{\frac{1}{4}}\right]$ (where $\mathrm{U}_{\mathrm{C}}$ and $\mathrm{H}_{\mathrm{dep}}$ are the pinning energy barrier and the domain wall depinning magnetic field, respectively) gives $\frac{U_{c}}{k_{B T}}\left(H_{\text {dep }}\right)^{\frac{1}{4}}=133(O e)^{\frac{1}{4}}$.

Hysteresis loops taken with and without the SAW indicate that the effects of the SAW driven strain are to dramatically decrease the coercive field. As seen in Fig. 2, this is a sharply resonant effect, with a linear dependence on strain (since the strain amplitude is proportional to the applied voltage), with a slope of $-3.5 \mathrm{G} / \mathrm{V}$. If we assume coherent rotation, a simple Stoner Wohlfarth model estimate results in a decrease in the out-of-plane anisotropy of $252 \mathrm{~J} / \mathrm{m}^{3}$ per Volt. Note that the change in coercivity seen here differs in a fundamental way from previously reported experiments, ${ }^{11}$ in which DC tensile (compressive) strain decreased (increased) the anisotropy linearly with $\varepsilon$. Here, we rapidly alternate between compressive and tensile strain, at approximately $10^{8}$ times a second, and the changes in coercivity are proportional to the magnitude of this strain, $|\varepsilon|$. SAW induced coercivity changes have been seen in highly magneto-strictive galfenol $^{12}$ as well as in the dilute magnetic semiconductor $(\mathrm{Ga}, \mathrm{Mn})(\mathrm{As}, \mathrm{P}) .{ }^{13}$ In the former, ${ }^{12}$ in addition to the average reduction in Hc, microscopy measurements indicate a spatially varying $\mathrm{Hc}$, with the largest changes occurring at the antinodes of a two dimensional standing wave. The lowering of $\mathrm{Hc}$ at the resonance frequency in our sample is also starkly visible in spatial maps showing the coercive field as a function of position, with and without SAW. In the absence of SAW, Hc is quite uniform, with only small variations around the edges of the image. With the SAW turned on, two effects manifest themselves: the average value of $\mathrm{Hc}$ decreases quite substantially, but there is also a large spatial variation, which does not appear to be related to any SAW generated standing wave patterns.

The effects of the SAW on domain wall velocity are clearly seen in a movie of domain wall motion with and without SAW (see supplementary material S-2), showing a rapid motion of domain walls across the sample surface with the SAW on. We have quantified this velocity as a function of SAW frequency and amplitude in a number of different ways. First, we measure the average velocity of a domain wall, using a method similar to that described above. We first saturate the sample and then apply $1 \mathrm{~s}$ pulses of an oppositely directed field of $134 \mathrm{G}$, resulting in the appearance of reverse domains. The reverse domains were moved by applying $1 \mathrm{~s}$ pulses of $115.5 \mathrm{G}$. The average velocity of the moving domain wall is shown in Fig. 3(a) as a function of applied power for different frequencies, including the resonant frequency of $96.6 \mathrm{MHz}$. Clearly, the resonant strain wave produces a significant change in the domain wall velocity that increases with excitation voltage. The sharply resonant effect rules out heating as a driver for the increased domain wall velocity since previous experiments of heating as a function
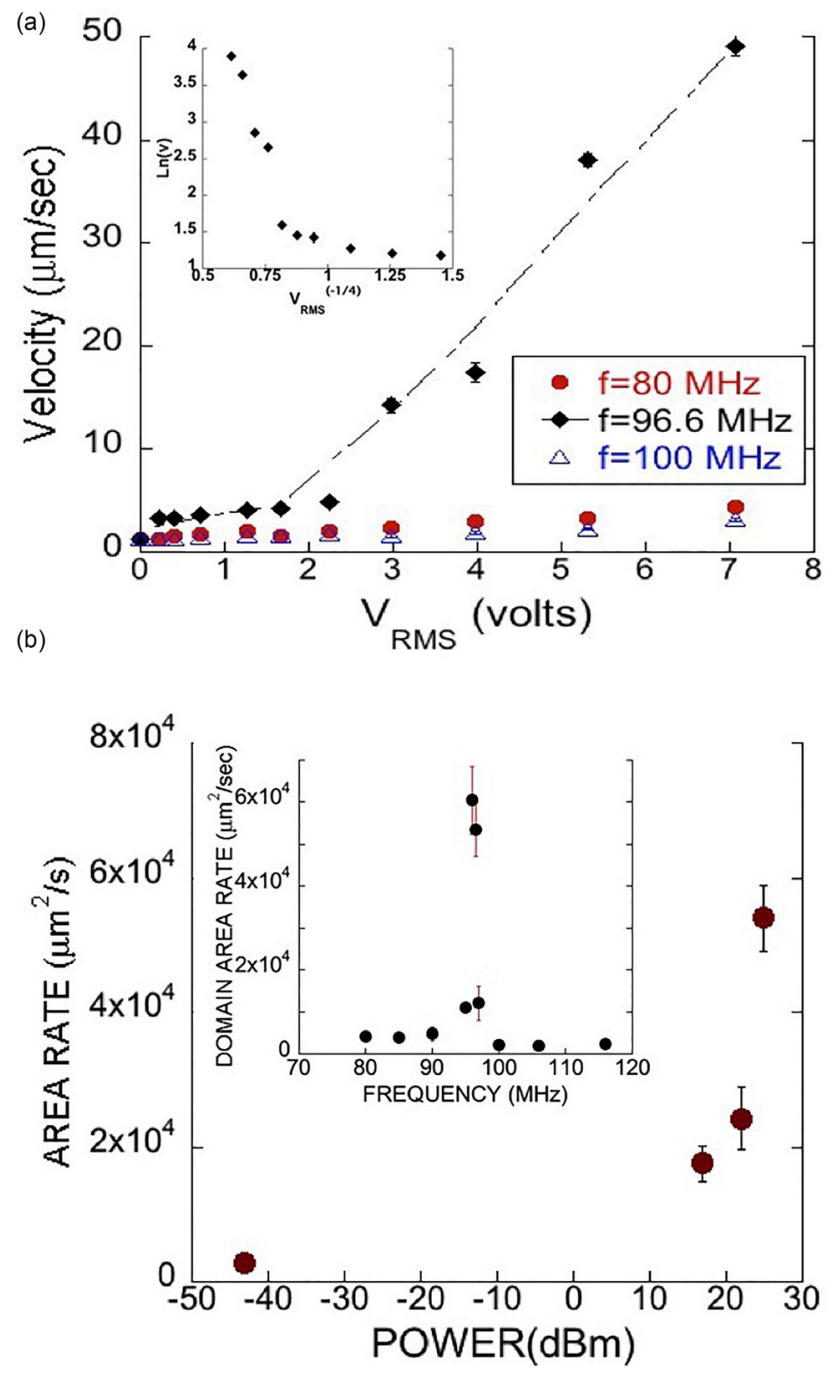

FIG. 3. (a) Domain velocity as a function of applied voltage at three different frequencies. (Inset) A plot of Ln (velocity) vs. $\mathrm{V}^{-1 / 4}$ showing clear deviations from a straight line at higher voltages. (b) Plots of the rate of change of domain area, $\mathrm{dA} / \mathrm{dt}$, as a function of applied power. The inset shows the frequency dependence of this rate change, which peaks sharply at the resonant frequency. 
of frequency at an input power of $25 \mathrm{dBm}$ (Ref. 14) indicate that the temperature rise at resonance is less than $10 \mathrm{~K}$ higher than that of resonance. From the fit to Fig. S-1 (supplementary material), viz. $\frac{U_{c}}{k_{B S}}\left(H_{d e p}\right)^{\frac{1}{4}}=133(O e)^{\frac{1}{4}}$, we calculate that at a $\mathrm{B}$ field value of $115 \mathrm{Oe}$, this temperature change would result in a $\sim 10 \%$ change in the domain wall velocity.

The effective field term for strain driven domain wall motion is proportional to the strain $\varepsilon$, which in turn is linearly dependent on the applied voltage. A plot of $\operatorname{Ln}$ (velocity) vs. $\mathrm{V}_{\mathrm{RMS}}{ }^{-1 / 4}$ (inset) shows clear deviations from linearity with increasing $\mathrm{V}_{\mathrm{RMS}}$, an indication that the strain driven domain wall motion transitions away from the creep regime. Second, we measure the rate at which the domain area changes [Fig. 3(b)] as a function of SAW frequency and power, at a constant field of $115 \mathrm{G}$. There is a 15 -fold increase in this rate at the resonance frequency and a monotonic increase with power.

The data in Fig. 3(a) were the average velocities obtained by averaging both across the domain front (roughly parallel to the y-axis) and in time as the domain wall progressed across the $\mathrm{x}$-axis. This averaging procedure does not take into account pinning/depinning sites that alter the progress of the domain wall. To observe the spatial modulation in domain wall velocity that was simulated in Ref. 4, we initially investigated the velocity of individual strips of the domain wall, i.e., those with the same y-axis coordinate. The variations measured cannot be ascribed to any period corresponding to the SAW standing wave. We attribute this to (i) an inability to exclude pinning sites, (ii) lack of control over the x-positions of the domain wall, and (iii) limitations in the upper and lower range of domain velocities we can measure.

To bypass these limitations, we have measured domain images as a function of time at a steady, DC field value. The image in Fig. 4(a) shows the time it takes for a domain to switch from up to down, with the domain wall moving from left to right, at a fixed field value of $115.4 \mathrm{G}$ (after saturation) and a SAW power of $25 \mathrm{dBm}$. The upper panel of Fig. 4(b) shows a single pixel cross-sectional line of a domain wall moving from right $(\mathrm{x}=0)$ to left (starting at the opposite end of the $\mathrm{Co} / \mathrm{Pt}$ rectangle) for two frequencies of applied SAWs, at resonance and far from resonance $(80 \mathrm{MHz})$. The vertical axis indicates the time it takes for a particular pixel to change from up to down. The data obtained at $80 \mathrm{MHz}$ show a steady increase in the time for pixel switching as the domain wall sweeps from right to left, corresponding to a nearly constant velocity, whereas at resonance, there are (almost) flat plateaus in time as a function of $\mathrm{x}$, indicating a very fast velocity, interspersed with abrupt jumps corresponding to a much slower velocity. The derivative of this dataset, $\Delta \mathrm{t} / \Delta \mathrm{x}$, shows a series of abrupt jumps at intervals of $10 \mu \mathrm{m}$, indicating regions in which the velocity drops sharply. These regions of abruptly slowed velocity correspond to the nodes and antinodes of the standing waves.

Previous micromagnetic simulations ${ }^{4}$ of thin films with in-plane magnetization with longitudinal strain indicate that the periodic decrease in the domain wall velocity should correspond only to antinodes of the standing wave. We have performed micromagnetic simulations of a thin magnetic nanowire (since simulating a thin film would be computationally demanding) with out of plane anisotropy and

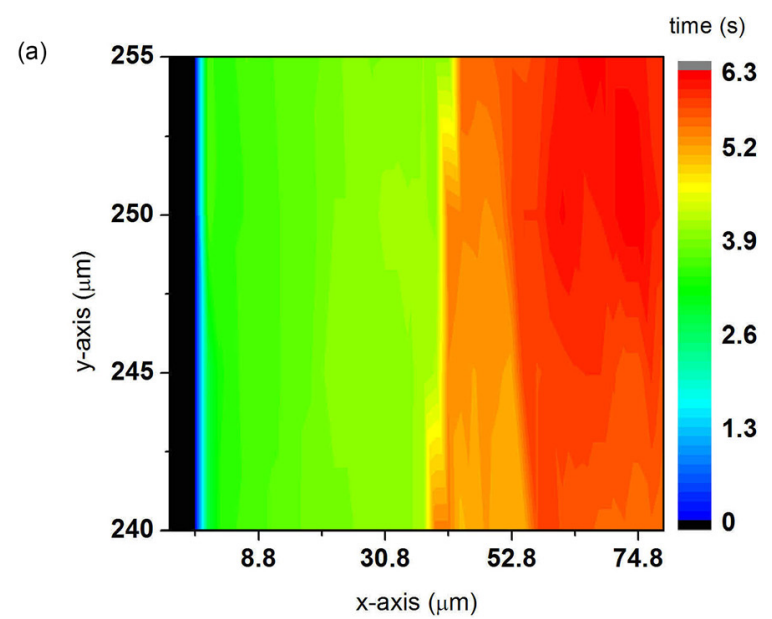

(b)

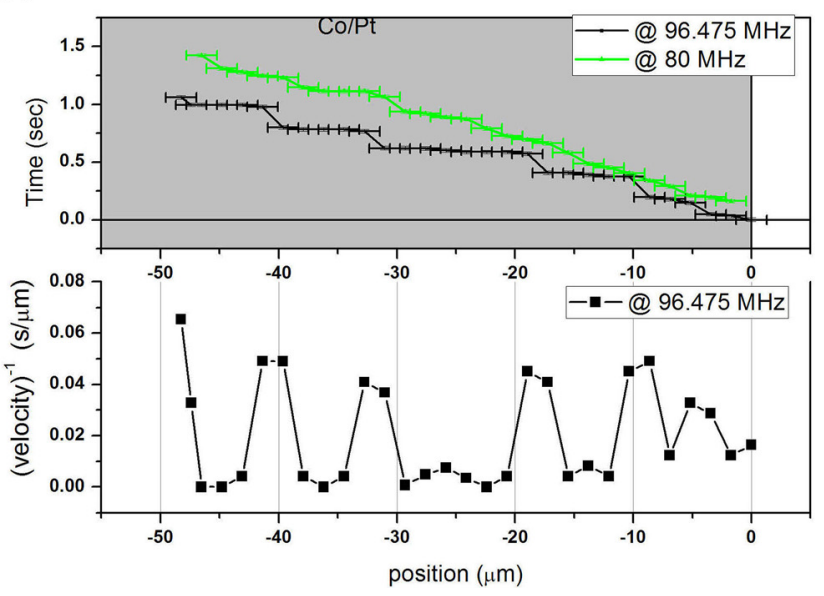

FIG. 4. (a) A two-dimensional spatial map of a domain wall moving from left to right, where the spectrum represents the time of switching of each pixel. There are regions of almost constant time (for example, the wide band of green) in which the domain wall progresses faster than the time resolution (b) Upper panel: The switching time as a function of x-position for a narrow strip of $\mathrm{Co} / \mathrm{Pt}$, for a domain wall moving from right to left. At the off resonance condition, the time increases linearly with the distance, indicating almost constant domain wall velocity. At resonance, the switching time is lowered, corresponding to a faster average velocity, and shows significant differences as a function of position. Lower panel: $\Delta \mathrm{t} / \Delta \mathrm{x}$ obtained from the black data as a function of $x$, showing regions of very slow velocity (peaks) with a periodicity of $10 \mu \mathrm{m}$, separated by regions of very fast velocities.

counter-propagating SAW with both $\varepsilon_{\mathrm{xx}}$ and $\varepsilon_{\mathrm{xy}}$ strain components, assuming a Neel domain wall through the thickness of the film. The results are similar to those in Ref. 4, indicating a translation of the DW from a node to an antinode, with maximum velocities occurring at the nodes of the standing wave. This is expected because the only relevant strain driving term for a Neel wall arises from the longitudinal standing wave $\varepsilon_{\mathrm{xx}}$. The $10 \mu \mathrm{m}$ periodicity in the domain wall velocity that appears in our data may arise from a more complex standing wave pattern. Reflections from the edges of the $\mathrm{LiNbO}_{3}$ wafer could set up a complex pattern of standing waves, but we discount this for two reasons. The distance to the edges is much greater than the distance between the two IDTS (on the order of centimeters compared to the $0.4 \mathrm{~mm}$ distance between the IDTs), and the edges are neither parallel nor straight. We conjecture that the transverse strain standing wave, although weak, may drive domain walls at the antinodes of the longitudinal standing wave, towards the antinodes of the transverse $\varepsilon_{x y}$ standing waves, which 
correspond to the nodes of the longitudinal standing wave. Hence, the combined effects of the longitudinal and transverse standing waves on the domain wall is to drive domain walls towards the antinodes of both waves, separated by a spacing of $10 \mu \mathrm{m}$.

In conclusion, we have shown that SAWs are efficient drivers of domain wall motion, as evidenced by the rapid increase in domain wall velocities with the increasing strain amplitude. Note that the fastest domain wall velocities we measure are limited by purely experimental constraints that include the inductance of the magnet and the capture frame rate of the camera. The effect is highly resonant, occurring only in a narrow range of frequencies, and we have clear evidence for a spatial modulation of the velocity, indicating pinning of the domains at a spacing of $\lambda / 4$. We attribute this to the combined effect of longitudinal and transverse strain standing waves.

See supplementary material for domain velocity as a function of amplitude of the $1 \mathrm{~s}$ field pulse [as shown in Fig. S-1; inset: $\mathrm{Ln}$ (velocity) vs $\mathrm{H}^{(-1 / 4)}$ ]. The straight line fit verifies that these field amplitudes correspond to the creep regime.

Movies of domain wall motion without (Movie S2-1) and with (Movie S2-2) a SAW. The magnetic field applied is 115.4 Oe for both cases, and the voltage to the SAW is $\mathrm{V}_{\mathrm{RMS}}=4.46 \mathrm{~V}$.

This work was supported by NSF (DMR-1409622) and NSF Nebraska MRSEC (DMR-1420645). Maya Abo
Dominguez and James Rehwalt Alexander were supported by the J. A. Woollam Foundation, the Nebraska Center for Materials and Nanoscience, and the University of NebraskaLincoln.

${ }^{1}$ G. S. D. Beach, C. Nistor, C. Knutson, M. Tsoi, and J. L. Erskine, Nat. Mater. 4, 741 (2005).

${ }^{2}$ M. Yamanouchi, D. Chiba, F. Matsukura, and H. Ohno, Nature 428, 539 (2004).

${ }^{3}$ S.-H. Yang, K.-S. Ryu, and S. Parkin, Nat. Nanotechnol. 10, 221 (2015).

${ }^{4}$ J. Dean, M. T. Bryan, J. D. Cooper, A. Virbule, J. E. Cunningham, and T. J. Hayward, Appl. Phys. Lett. 107, 142405 (2015).

${ }^{5}$ U. Singh and S. Adenwalla, Nanotechnology 26, 255707 (2015).

${ }^{6}$ S. Davis, A. Baruth, and S. Adenwalla, Appl. Phys. Lett. 97, 232507 (2010).

${ }^{7}$ R. Ploessl, J. N. Chapman, M. R. Scheinfein, J. L. Blue, M. Mansuripur, and H. Hoffmann, J. Appl. Phys. 74, 7431 (1993).

${ }^{8}$ D. Navas, C. Redondo, G. A. Badini Confalonieri, F. Batallan, A. Devishvili, Ó. Iglesias-Freire, A. Asenjo, C. A. Ross, and B. P. Toperverg, Phys. Rev. B 90, 054425 (2014).

${ }^{9}$ See https://imagej.nih.gov/ij/ for details reading the ImageJ software.

${ }^{10}$ P. J. Metaxas, J. P. Jamet, A. Mougin, M. Cormier, J. Ferre, V. Baltz, B. Rodmacq, B. Dieny, and R. L. Stamps, Phys. Rev. Lett. 99, 217208 (2007).

${ }^{11}$ P. M. Shepley, A. W. Rushforth, M. Wang, G. Burnell, and T. A. Moore, Sci. Rep. 5, 7921 (2015).

${ }^{12}$ W. Li, B. Buford, A. Jander, and P. Dhagat, IEEE Trans. Magn. 50, 3100704 (2014).

${ }^{13}$ L. Thevenard, I. S. Camara, J.-Y. Prieur, P. Rovillain, A. Lemattre, C. Gourdon, and J.-Y. Duquesne, Phys. Rev. B 93, 140405(R) (2016).

${ }^{14}$ C. Huck, H. P. Zidek, T. Ebner, K. C. Wagner, and A. Wixfort, in 2013 IEEE International Ultrasonics Symposium (IUS), Prague, 21-25 July 2013. 